\title{
Entsteht ein Markt für Unternehmenskontrolle? Der Fall Mannesmann
}

\section{Article by an MPIfG researcher}

Martin Höpner, Gregory Jackson: Entsteht ein Markt für Unternehmenskontrolle? Der Fall Mannesmann.

In: Leviathan 29(4), 544-563 (2001). VS Verlag für Sozialwissenschaften

The original publication is available at the publisher's web site: http://dx.doi.org/10.1007/s11578-001-0034-6

\section{Einleitung}

Die erfolgreiche feindliche Übernahme der Mannesmann AG durch VodafoneAirtouch (nachfolgend: Vodafone) markiert einen Wendepunkt in der deutschen Wirtschaftsgeschichte, dem schleichende Veränderungen in den 1990er Jahren zu Grunde liegen: Das deutsche Corporate Governance System hat sich in einer Weise entwickelt, dass feindliche Übernahmen möglich wurden.

Kapitalistische Systeme zeichnen sich durch den freien Austausch von Waren auf Märkten aus. Märkte sind eingebettet in soziale Institutionen, die regulieren, was eine Ware ist und also „frei" gehandelt werden darf. Ein in diesem Sinne freier Markt ist der Produktmarkt, allerdings nur in räumlichen Grenzen (Binnenmarkt nach innen, Zollschranken nach außen) und in sachlichen Grenzen (Fernseher dürfen frei gehandelt werden, Lebensmittel manchmal, elektrischer Strom neuerdings auch, Plutonium nie). Mit der Entstehung von Märkten fïr Arbeitskräfte entsteht der Kapitalismus im eigentlichen Sinne; die soziale Einbettung von Märkten zeigt sich allerdings nirgends so deutlich wie in der starken Regulierung der Arbeitsmärkte und in der sozialen Abfederung der Risiken, die mit dem Handel der Ware Arbeitskraft verbunden sind. Der freie Kapitalmarkt erscheint als natürliches Merkmal kapitalistischer Systeme, gleichwohl ist die Liberalisierung der Kapitalmärkte in den 70er Jahren in der Geschichte des Kapitalismus in Deutschland ein junges Ereignis. Der Handel mit riskanten Finanzprodukten (z.B. Termingeschäfte) und der Umgang mit Finanzrisiken bleibt Gegenstand staatlicher Regulierung. Mit dem Markt fiir Unternehmenskontrolle hat sich schließlich ein vierter abgrenzbarer Markt ausdifferenziert, auf dem Unternehmensanteile in einer Größe gehandelt werden, die die Ausübung von Macht über Unternehmen erlauben. Damit werden Unternehmen, die Waren produzieren, selbst zu Waren (Windolf 1994, S. 81 f.). Im internationalen Vergleich werden Corporate Governance Systeme danach klassifiziert, ob sie feindliche Übernahmen erlauben oder nicht (de Jong 1992, 1997).

Warum kommt feindlichen Übernahmen eine solch herausragende Rolle zu? Warum hat es sie in der Bundesrepublik bis dato nicht gegeben? Und welche 
Veränderungen führten dazu, dass der Fall Mannesmann möglich wurde? Mit diesen Fragen beschäftigt sich die vorliegende Fallstudie.

\section{Der Markt für Unternehmenskontrolle}

Die ökonomische Bedeutung des Markts für Unternehmenskontrolle wurde in der "Agency-Theorie" herausgearbeitet. Sie beschäftigt sich mit folgender Problemstellung: Wie können Aktionäre als Auftraggeber (principals) sicherstellen, dass die von ihnen mit der Verwaltung ihres Eigentums beauftragten Manager (agents) tatsächlich im Sinne der Anteilseigner handeln? In ihrer bahnbrechenden Studie von 1932 hatten Adolph A. Berle und Gardiner C. Means gezeigt, dass in $44 \%$ der von ihnen untersuchten amerikanischen Unternehmen die Anteile derart breit gestreut waren, dass von einer „Herrschaft der Manager" über diese Unternehmen gesprochen werden musste. In nur 11\% der Unternehmen gab es eindeutige Mehrheitsaktionäre. Aufgrund des von ihnen beobachteten Trends zur breiten Streuung des Eigentums an Unternehmen gingen sie von einer historischen Entwicklung zur Trennung von Eigentum und Kontrolle aus. Minderheitsaktionäre mit diversifizierten Portfolios neigen dazu, sich nur selten in die Unternehmenspolitik einzumischen, nur in begrenztem Maße spezielle Unternehmensinformationen zu sammeln und auf sinkende Rentabilität mit dem Verkauf ihrer Anteile zu reagieren. Damit entstehen Freiräume für Manager, ihre eigenen Interessen - zum Beispiel die Investition in unrentable Prestigeobjekte - zu verfolgen.

Verschiedene Mechanismen zur Reduzierung der Agency-Kosten wurden daraufhin in der ökonomischen Theorie diskutiert: Der staatliche Schutz von Minderheitsaktionären, anreizkompatible Verträge zwischen Managern und Auftraggebern, die Konzentration der Aktionärsstrukturen zu Aktienblocks mit der Fähigkeit zu einem effektiven Monitoring und Märkte für Unternehmenskontrolle (Shleifer/Vishny 1996).

Die These von der disziplinierenden, die Herrschaft der Manager begrenzenden Wirkung des Markts für Unternehmenskontrolle wurde zuerst von Henry Manne (Manne 1965, S. 113) beschrieben: „The lower the stock price, relative to what it could be with more efficient management, the more attractive the take-over becomes to those who believe that they can manage the company more efficiently." Manne ging von einer engen Beziehung zwischen den Leistungen der Managements und der Entwicklung der Aktienkurse aus. Die Existenz eines Markts für Unternehmenskontrolle müsste unter diesen Bedingungen eine überwachende, die Agency-Kosten senkende Wirkungen entfalten. Damit beschrieb Manne einen Regelungsmechanismus zur Kopplung der Managerinteressen an die Interessen der Anteilseigner, der mit der Präferenz der Kleinanleger nach Liquidität (exit statt voice) kompatibel ist. Schon die theoretische Möglichkeit einer feindlichen Übernahme 
müsste, Mannes Theorie zufolge, Manager zu effizientem Umgang mit den ihnen zur Verfügung gestellten Ressourcen anhalten.

Allerdings fallen die Ergebnisse der empirischen Untersuchungen zu feindlichen Übernahmen in den USA weniger eindeutig aus, als Manne es mit seinem innovativem Modell formuliert hatte. In den 1960er Jahren fand in amerikanischen Unternehmen ein starker Trend zur Diversifizierung der Aktivitäten statt, dessen Ergebnisse in der Folgezeit aber enttäuschten. Diversifizierte Unternehmen wurden an der Börse mit einem Abschlag, dem so genannten "Conglomerate Discount" gehandelt. Eine Lockerung der Anti-Trust-Gesetzgebung durch die Reagan-Administration und eine Innovation auf den Finanzmärkten - die ,junk bonds", die amerikanischen Raidern große Mengen an Risikokapital für feindliche Übernahmen zur Verfügung stellten - führten zu der beispiellosen Übernahmewelle der 1980er Jahre, deren Ergebnis die De-Diversifizierung der amerikanischen Konglomerate war. Konglomerate wurden aufgekauft, zerschlagen und in Einzelteilen veräußert (Bhagat/Shleifer/Vishney 1990; Davis/Diekmann/Tinsley 1994). Nach den 1980er Jahren ebbte die Übernahmewelle merklich ab. Zwischen 1990 und 1996 wurden nur 23 der Unternehmen aus den Fortune 500 das Ziel von Übernahmeangeboten, und nur 6 dieser 23 Angebote wurden als feindlich klassifiziert (Davis/Robbins 2001).

Hinsichtlich der Rentabilitätseffekte der Übernahmewelle kommt die wissenschaftliche Literatur zu ambivalenten Ergebnissen (Beyer 1998, S. 70-78). Eindeutige Nachweise von schwacher Leistung von Unternehmen vor deren feindlicher Übernahme konnten nicht erbracht werden (Davis/Stout 1992; Franks/Mayer 1995). Die Aktionäre der Zielunternehmen scheinen durch die Übernahmeprämien zu gewinnen (Bittlingmayer 1998, S. 28). Ein Wertzuwachs für die Aktionäre der Bietergesellschaften scheint allerdings nur schwer nachzuweisen zu sein (Gerke/ Garz/Oerke 1995). Eine Studie über feindliche Übernahmen in Großbritannien kommt zu dem Ergebnis, dass die Produktivität von potenziellen Zielgesellschaften kaum gesteigert wurde, aber das Investitionsniveau gesenkt und die Dividenden gesteigert wurden (Nuttall 1999).

Insgesamt ist die Theorie der feindlichen Übernahmen als „Effizienzmaschinerie" mit Vorsicht zu genießen. Die Umverteilungswirkungen von feindlichen Übernahmen sind eindeutiger als die Effizienzwirkungen. So formulierte Henry Manne (1965): „Given the fact of special tax treatment for capital gains, we can see how this mechanism for taking control of badly run corporations is one of the most important 'get rich-quick' opportunities in our economy today."

Der niederländische Ökonom Henk de Jong (1997) hat gezeigt, dass in Corporate Governance Systems mit feindlichen Übernahmen größere Teile der Nettowertschöpfung an die Anteilseigner ausgeschüttet werden, während die bei de Jong als "germanisch" bezeichnete Ländergruppe durch eine starke Verteilungsposition der Arbeitnehmer und einen hohen Anteil an Gewinnen, die zur Reinvestition im Unternehmen verbleiben, gekennzeichnet ist. Andrei Shleifer und 
Lawrence H. Summers (Shleifer/Summers 1988) haben die These aufgestellt, dass die Aktionärsgewinne bei feindlichen Übernahmen zu einem großen Teil nicht aus gesteigerter Rentabilität, sondern aus dem Bruch von impliziten Verträgen mit den Stakeholdergruppen - vor allem den Abnehmern und den Beschäftigten resultieren und damit das Vertrauen zwischen ihnen unterminieren.

Drei weitere Argumente nähren zusätzliche Zweifel an der Effizienzthese: Erstens erscheint die Annahme, dass die Kapitalmärkte Unternehmen stets zu ihrem „fairen“ Preis bewerten, zweifelhaft (Kraakman 1988). Zweitens haben feindliche Übernahmen nicht nur eine begrenzende, sondern auch eine opportunistisches Verhalten von Managern ermöglichende Wirkung, weil die Fähigkeit zu feindlichen Übernahmen wiederum als Instrument im Ausbau des Machtbereich der übernehmenden Manager dienen kann. Und drittens erscheint es äußerst fragwürdig, ob die nach feindlichen Übernahmeofferten von den Managern der Zielunternehmen eingeleiteten Verteidigungsmaßnahmen - von der schnellen Verlängerung von Vorstandsverträgen über die beispielsweise bei Mannesmann erfolgte Vereinbarung horrender Ablösesummen bis hin zum Ankauf unattraktiver Unternehmensteile - einer Ökonomie gut tun können.

Bis in die 1990er Jahre hinein war Deutschland durch eine niedrige Aktivität bei Fusionen und Übernahmen gekennzeichnet (Dietrich 1994), wobei die Transaktionen in der Regel ${ }^{1}$ als freundliche Übereinkünfte, nicht als feindliche Übernahmen organisiert wurden. In der "Varieties of Capitalism"-Literatur wurde das Fehlen feindlicher Übernahmen als institutionelle Voraussetzung des koordinierten, langfristig orientierten, auf inkrementale Innovation und Qualitätsproduktion setzenden, rapide Kurswechsel erschwerenden und durch die Machtbalance zwischen Stakeholder-Gruppen gekennzeichneten Typs des Kapitalismus diskutiert (Hall/ Soskice 2001; Albert 1992; Streeck 1992b; de Jong 1992, 1997; Jackson 2001). In dieser Perspektive können Barrieren gegen feindliche Übernahmen als „beneficial constraints" (Streeck 1997) interpretiert werden, die vor dem Bruch impliziter Verträge bewahren. Mehrere voneinander unabhängige Faktoren können für das Fehlen feindlicher Übernahmen im „Modell Deutschland“ verantwortlich gemacht werden:

1. Die Eigentümerstrukturen deutscher Unternehmen sind durch eine starke Konzentration des Aktienbesitzes gekennzeichnet. Im Jahr 1998 befanden sich nur 22 der 100 größten deutschen Unternehmen in mehrheitlichem Streubesitz,

1 Der Fall Mannesmann war das erste offene, direkt an die Aktionäre gerichtete feindliche Übernahmeangebot in Deutschland. Allerdings ist diskutabel, auch den im Dissens mit Managern der Zielgesellschaften stattfindenden Aufkauf von Aktienpaketen durch andere Unternehmen als eine „weiche Form" feindlicher Übernahmen zu werten. Jenkinson/Ljungvist (1999) zählen in Deutschland 17 Fälle des Aufbaus "unfreundlicher" Aktienpakete zwischen 1988 und 1996. Die Versuche, Continental (1990-1993) und Thyssen (1997) feindlich zu übernehmen, werden im Folgenden als kontrastierende Fälle zur Auseinandersetzung zwischen Mannesmann und Vodafone genutzt. 
und nur bei 11 dieser Unternehmen waren mindestens $75 \%$ der Aktien breit gestreut (Monopolkommission 2000, S. 259-265). Ein Abgleich mit früheren Studien über Eigentümerstrukturen deutscher Großunternehmen zeigt keine deutlichen Unterschiede dieser Aktionärsverhältnisse gegenüber den vergangenen Jahrzehnten (Monopolkommission 1980). Nimmt man an, dass feindliche Übernahmen ab einer Schwelle von etwa 75\% Streubesitz denkbar sind, beträgt der Anteil der theoretisch übernehmbaren Großunternehmen in Deutschland also nicht mehr als gut $10 \%$.

2. Als entscheidende Verhinderer feindlicher Übernahmen in Deutschland können die Großbanken angesehen werden, die in ihrer multifunktionalen Stellung als Kreditgeber, Aktienhalter, Verwalter der Depotstimmen und Unternehmensaufseher über mehrere Jahrzehnte eine zentrale industriepolitische Rolle spielten. Auch wo Großbanken nicht als Aktienhalter auftreten, spielen sie als Verwalter der Depotstimmen eine entscheidende Rolle und erlangen in Aktiengesellschaften mit gestreuter Besitzstruktur häufig die mittelbare Stimmenmehrheit auf Hauptversammlungen (Baums/Fraune 1995). Im Monitoring von Großunternehmen spielen Banken eine zentrale Rolle. In nahezu allen Aufsichtsräten der großen Industrie- und Handelsunternehmen sitzen ein bis drei, in Ausnahmefällen bis zu fünf Bankenvertreter. Anfang der 1990er Jahre waren um die $40 \%$ der Aufsichtsratsvorsitzenden der 40 größten börsennotierten Unternehmen aus Industrie und Handel Bankenvertreter. In den 1990er Jahren setzte ein rapider Wandel in der industriepolitischen Rolle deutscher Großbanken ein, die sich aus dem Monitoring von Industrieunternehmen zurückziehen und ihre Zukunft im Investmentbanking sehen.

3. Als "Giftpille" gegen potenzielle feindliche Übernehmer wurde auch die Mitbestimmung angesehen. Die Beteiligung von Arbeitnehmern im Aufsichtsrat mindert den direkten Einfluss der Anteilseigner. Zwar führt das Doppelstimmrecht des Aufsichtsratsvorsitzenden dazu, dass die Arbeitnehmerbank im Konfliktfall überstimmt werden kann. Kampfabstimmungen im Aufsichtsrat sind allerdings selten, und gibt es Meinungsverschiedenheiten auf der Seite der Anteilseigner, kann die Arbeitnehmerbank durch die Unterstützung einer der beiden Seiten starken Einfluss gewinnen. Eine besonders starke Begrenzung der Macht der Eigentümer besteht in montanmitbestimmten Unternehmen, wo ein feindlicher Übernehmer gegen den Willen des neutralen, von beiden Seiten bestimmten 21. Aufsichtsratsmitglieds keinen Vorstand austauschen kann.

4. Weitere Merkmale des deutschen Wirtschafts- und Aktienrechts wirken hemmend auf potenzielle Übernehmer. Wo nach dem Handelsgesetzbuch (HGB) bilanziert wird, ist die wahre Werthaltigkeit von Unternehmen für Outsider nur schwer einzuschätzen. Bis in die späten 1990er Jahre erlaubte das deutsche 
Aktienrecht Stimmrechtsbeschränkungen, die die Ausübung von Stimmrechten auf Hauptversammlungen ab einer definierten Schwelle - häufig 5\% - verunmöglichten und damit große Aktienpakete „unerwünschter" Anteilseigner zu reinen Finanzanlagen statt zu Instrumenten der Ausübung von Macht über Unternehmen werden ließen. Auch Bestimmungen des deutschen Aktiengesetzes, denen zufolge bei der Abberufung von Aufsichtsratsmitgliedern, Kapitalerhöhungen und Kapitalherabsetzungen Hauptversammlungsmehrheiten von $75 \%$ notwendig sind, wirken erschwerend auf feindliche Übernahmen.

In den nachfolgenden Abschnitten stellen wir dar, warum die Übernahme von Mannesmann durch Vodafone trotz der oben beschriebenen Restriktionen möglich wurde.

\section{Der Fall Mannesmann ${ }^{2}$}

Konzernstruktur und Shareholder Value

Mannesmann war ein traditionsreicher Mischkonzern, der 1890 zur Röhrenproduktion gegründet wurde und sich Anfang des 20. Jahrhunderts in die Bereiche Stahl und Bergbau diversifizierte. In den frühen 1970er Jahren erfolgte der Strukturwandel vom Montan- zum Maschinenbauunternehmen. In den 1980er Jahren wurde der Bereich Automobilzubehör in den Konzern integriert.

Den radikalsten Wandel vollzog Mannesmann in den 1990er Jahren: Im Zuge der Liberalisierung des deutschen Telekommunikationsmarkts baute Mannesmann das Mobilfunknetz D2 auf, erwarb Mehrheitsbeteiligungen von in- und ausländischen Telekommunikationsunternehmen und wurde zu einem der wichtigsten europäischen Wettbewerber neben Vodafone, British Telekom, France Télécom, der niederländischen KPN und der Deutschen Telekom. ${ }^{3}$ Im Zeitraum von 1990 bis zum 1. Halbjahr 1999 entfielen zwei Drittel aller Investitionen auf diesen Bereich, während im Bereich Röhren de-investiert wurde.

Im Mai 1999 löste der promovierte Jurist Klaus Esser seinen Vorgänger Joachim

2 Wir möchten den Praktikern danken, die uns zwischen Herbst 1999 und Sommer 2000 für Interviews zur Verfügung standen: Gerd Kappelhoff, IG Metall, Konzernbetreuer von Thyssen-Krupp; Roland Köstler, Referatsleiter Recht im Mitbestimmungsförderungsbereich der Hans-Böckler-Stiftung und Mitglied des Mannesmann-Aufsichtsrats; Werner Nass, Gesamtbetriebsrat bei Thyssen-Krupp; Horst Urban, ehemaliger Vorstandsvorsitzender von Continental; Christoph W. Stein und Fabian Kirchmann, Leiter der Investor-Relations-Abteilung von Mannesmann; Rainer Schmidt, IG Metall, Konzernbetreuer von Mannesmann.

3 Vor der Übernahme bestand Mannesmann aus den Unternehmenssegmenten Telekommunikation (Mannesmann Mobilfunk/D2, Arcor, Omnitel. Infostrada und Orange), Maschinenbau/Automobilzulieferung (Rexroth, Dematic, Demag Krauss-Maffei, VDO und Sachs), Röhren und Luxusuhren. 
Funk als Vorstandsvorsitzenden ab. Im September 1999 kündigte Esser an, die klassischen Bereiche des Konzerns ausgliedern und die künftigen Aktivitäten auf die Telekommunikation beschränken zu wollen. Von institutionellen Investoren war dieser Schritt seit langem gefordert worden. Unmittelbar nach dem Teilungsbeschluss am 23. September 1999 stieg der Kurs der Mannesmann-Aktie um 7\%. Bei Mannesmann hatte man vermutet, der Kursanstieg nach der Ankündigung der Konzernteilung - interpretiert als Bereinigung um den „Conglomerate Discount", also den Abschlag im Börsenwert von Mischkonzernen - werde deutlicher ausfallen.

Mannesmann war eines der wenigen deutschen Unternehmen ohne Großaktionäre. Mitte 1999 wurden etwa 60\% der Anteile von institutionellen Anlegern gehalten, davon allein $40 \%$ bei ausländischen institutionellen Anlegern. ${ }^{4}$ Mannesmann konnte auf eine furiose Kursentwicklung in den späten 1990er Jahren zurückblicken. Wer 1996 eine Mannesmann-Aktie für 34 Euro erwarb, konnte sie im Februar 2000 für 300 Euro veräußern. Gleichwohl gehörte Mannesmann nicht zu den Vorreitern der "Shareholder Value-Bewegung" in Deutschland. Sehr gute Noten erhielt der Konzern für seine Investor-Relations-Arbeit, vergleichbar mit DaimlerChrysler und SAP. Die Rechnungslegung allerdings war intransparent und folgte den Regeln des HGB statt internationalen Bilanzierungsregeln. Auch was das Ausmaß betrifft, in dem die Managervergütung an die Aktienkursentwicklung gekoppelt war, sowie in Fragen der operativen Anwendung von Shareholder-Value-Kennziffern gehörte Mannesmann nicht zu den Vorreitern (Grafl Lenke/Schießer 1997, S. 19 ff.; Höpner 2001). Insgesamt verhielt sich das Management in den späten 1990er Jahren weniger kapitalmarktorientiert, als es aufgrund von Größe, Internationalität, zu 100\% gestreuter Eigentümerstruktur und Internationalität zu erwarten wäre. ${ }^{5}$ Der Wechsel des Vorstandsvorsitzes von Funk zu Esser markierte eine deutliche Ausweitung der Shareholder-Value-Orientierung von Mannesmann.

Übernahmegebot und dominante Abwehrstrategie

Das feindliche Übernahmeangebot an die Mannesmann-Aktionäre erfolgte nicht, weil ein schlecht geführtes Unternehmen an der Börse niedrig bewertet wurde und deshalb eine hohe Übernahmeprämie versprach. Der Übernahmeversuch war industriepolitisch motiviert. Vodafone und Mannesmann galten im Herbst 1999 als Verbündete. Im Gegensatz zu Mannesmann war Vodafone auf Mobilfunk fo-

4 Mit der Akquisition von Orange sank der Anteil des Streubesitzes von 100\% auf $89,8 \%$, weil der ehemalige Orange-Großaktionär Hutchison Whampoa nun 10,2\% der Anteile hiclt.

5 Die genannten Faktoren erweisen sich im Unternehmensvergleich als Determinanten der Kapitalmarktorientierung von Unternehmen (Höpner 2000a). 
kussiert und verfügte über keine Aktivitäten im Festnetz. Ende Oktober 1999 wurde das im November offiziell verkündete Mannesmann-Gebot für das britische Telekommunikationsunternehmen Orange bekannt. Diese Maßnahme wurde als Angriff Mannesmanns auf Vodafone in dessen heimischem Markt gewertet. Vodafone-Chef Chris Gent reagierte mit einem Mitte November unterbreiteten freundlichen, Anfang Dezember offiziell abgegebenen und direkt an die Aktionäre gerichteten feindlichen Übernahmeangebot. Es sah den Tausch von 53,7 VodafoneAnteilen pro Mannesmann-Aktie ohne Barkomponente vor. In der schriftlichen Begründung des Übernahmeangebots an die Mannesmann-Aktionäre setzte Vodafone im Wesentlichen auf die Größenvorteile eines gemeinsamen Konzerns (Vodafone 1999).

Hinsichtlich der Argumentationsstrategie bei Mannesmann sind zwei Linien zu unterscheiden (Mannesmann 2000): Erstens Argumente, die sich auf wahrscheinliche Wertsteigerungen bei alternativen Entwicklungsstrategien einer allein oder mit Vodafone operierenden Mannesmann AG bezogen, zweitens technische Argumente, die auf die Schwierigkeiten der Pläne Vodafones vor dem Hintergrund des deutschen Aktienrechts verwiesen. Dabei standen Verfahrensfragen zur kartellrechtlich notwendigen Abspaltung von Orange vor einer Übernahme von Mannesmann durch Vodafone im Vordergrund. Auffällig war, dass ein bestimmter Typus von Argumenten bei Esser keine Rolle spielte: Er stellte die grundsätzliche Legitimität des feindlichen Übernahmegebot Vodafones nicht in Frage. Die Verteidigungsstrategie Essers war auf die Gunst der Aktionäre ausgerichtet. In den veröffentlichten Stellungnahmen des Mannesmann-Vorstands fanden sich weder Hinweise auf drohende Arbeitsplatzverluste, noch auf die zu erwartende Erosion der Unternehmensmitbestimmung in der (nach der Fusion ausländischen) Konzernobergesellschaft. Damit steht die Verteidigungsstrategie Essers in scharfem Kontrast zu den Vorgängen um die Übernahmeversuche auf Continental (19901993) und Thyssen (1997), bei denen die Vorstandsvorsitzenden Urban und Vogel im Wesentlichen die Legitimität feindlicher Übernahmeverfahren mit ihrer Fokussierung auf den Nutzen der Anteilseigner erfolgreich verneinten (Vogel 1997; Baums 1993; Franks/Mayer 1998).

Als der italienische Reifenkonzern Pirelli in den frühen 1990er Jahren versuchte, Continental zu übernehmen - zu einem direkt an die Aktionäre gerichteten Angebot kam es dabei nicht -, konzentrierte sich ein wesentlicher Teil der Auseinandersetzungen auf die bei Continental geltenden Stimmrechtsbeschränkungen. ${ }^{6}$ Diese Abwehroption stand dem Mannesmann-Management nicht mehr zur Verfügung. Zwar galt bei Mannesmann eine Stimmrechtsbeschränkung, die besagte, dass keinem Aktionär auf der Hauptversammlung mehr als 5\% der Stimmen zukommen können; mit dem 1998 verabschiedeten Gesetz zur Kontrolle und Transparenz im Unternehmensbereich (KonTraG) wurden Stimmrechtsbeschränkungen

6 Eine detaillierte Beschreibung findet sich in Erker (1996). 
allerdings im Zuge der europäischen Harmonisierung mit einer Übergangsfrist bis Juli 2000 verboten.

Die Rolle von Betriebsräten und Gewerkschaften

Warum fiel der Protest von Betriebsrat und IG Metall gegen den Übernahmeversuch, im Vergleich zu den Vorgängen um Thyssen im Jahr 1997, so moderat aus? Zwei Gesichtspunkte sprachen gegen massiven Widerstand von Arbeitnehmerseite: Die Teilung des Konzerns war bereits vorher im Konsens mit, ja sogar auf Betreiben von Betriebsrat und Gewerkschaft vereinbart worden; und ein massiver Arbeitsplatzabbau war im Zuge einer Übernahme durch Vodafone nicht zu befürchten. Im Ergebnis wurden industriepolitische Forderungen zur Zukunft der Unternehmenssegmente ausgestellt, die Legitimität des Übernahmegebots von Gent aber nicht grundsätzlich in Frage gestellt.

Mannesmann zählte zu den Unternehmen mit besonders weitreichender Mitbestimmung. Der Grund dafür ist, dass Mannesmann in den 1950er Jahren in den Bereich des Montanmitbestimmungsgesetzes fiel. Seit über 20 Jahren versuchte man bei Mannesmann, sich der Anwendung des Montanmitbestimmungsgesetzes zu entziehen. Im Mai 1999 erklärte das Bundesverfassungsgericht die 1988 verabschiedete „Lex Mannesmann", die auf die Fortgeltung der Montanmitbestimmung bei Mannesmann zielte, ${ }^{7}$ für verfassungswidrig. Gleichwohl saß während des Übernahmekampfs mit Sigmar Sattler noch immer ein von der IG Metall bestellter Arbeitsdirektor im Konzernvorstand, was darauf hindeutet, dass die Auseinandersetzung um die Montanmitbestimmung bei Mannesmann eher symbolischer Natur war.

Als Klaus Esser die Shareholder-Value-Orientierung von Mannesmann vorantrieb, begrüßte die Arbeitnehmervertretung die Schritte in Richtung verstärkter Unternehmenstransparenz, weil auch sie von der verbesserten Informationslage profitierte. In der Ära von Essers Vorgänger Funk „mußten wir jedem Blatt hinterherrennen", das habe sich gebessert, so ein Mitglied der Arbeitnehmerbank im Mannesmann-Aufsichtsrat. Den Beobachter mag zunächst verwundern, dass die vor der feindlichen Übernahme bei Mannesmann beschlossene und von den Aktionären lange geforderte Konzernteilung nicht gegen den Mannesmann-Betriebsrat und die Arbeitnehmerbank im Aufsichtsrat durchgesetzt werden musste, sondern von der Arbeitnehmerseite sogar eingefordert und vorangetrieben wurde. Es sei datan erinnert, dass sich die Mannesmann AG 1999 als extrem heterogener Mischkonzern darstellte. Nach und nach hatte sich der Telekommunikationsbereich von Mannesmann zum wichtigsten und mit Abstand investitionsstärksten Segment

7 Nach dieser Gesetzesänderung galten die Bestimmungen des Montanmitbestimmungsgesetzes für Unternehmen mit einem Montan-Anteil (Eisen, Kohle, Stahl) ab 20\% des Umsatzes (vorher 50\%). 
entwickelt. In Analystenbesprechungen, berichten Mitarbeiter der Investor-Relations-Abteilung, ging es zu 90\% um den Telekommunikationsbereich. Ein Mitbestimmungsträger von Mannesmann beschreibt die damalige Situation wie folgt: „Die Entwicklung des Telekommunikationsbereichs wurde für die anderen Bereiche allmählich gefährlich. Während man in der Telekommunikation viele Milliarden Euro für die Akquisition von Orange ausgab, mußten wir in den klassischen Bereichen um jeden Hammer kämpfen."

Die klassischen Bereiche - Engineering, Automobilzubehör, Röhren - liefen Gefahr, im Wettbewerb um Investitionen und Managementkapazitäten dauerhaft benachteiligt $\mathrm{zu}$ werden. Interessenvertreter der Beschäftigten kritisierten die blockierte Globalisierung von VDO und Sachs, denn für Automobilzulieferer ist es überlebenswichtig, mit der Internationalisierung der Automobilunternehmen Schritt halten und vor Ort liefern zu können. Die Arbeitnehmervertretung trieb den Plan der Konzernteilung 1999 voran, um den älteren Segmenten eine eigene „ungestörte" Entwicklung zu ermöglichen. Auch der Telekommunikationsbereich sah sich durch die Unternehmensstruktur gehemmt: Durch den Conglomerate Discount wurden Akquisitionen verteuert. So erklärt sich, dass die Teilung der Unternehmensbereiche von Aktionären und Arbeitnehmervertretung gleichermaßen befürwortet wurde.

Die Segmente der Mannesmann AG stellten sich 1999 als gesunde, überlebensfähige Unternehmen dar. Anders als während des Übernahmekampfs zwischen Thyssen und Krupp im Jahr 1997 waren massive Arbeitsplatzverluste nicht zu erwarten. Im Telekommunikationsbereich war aus damaliger Perspektive der weitere Aufbau, kein Abbau von Kapazitäten in Sicht.

Gleichwohl konnte über die Absichten des potenziellen Übernehmers keine Klarheit bestehen. Betriebsrat und Gewerkschaften wiesen den Übernahmeversuch von Vodafone zurück und sprachen sich für eine eigenständige Entwicklung der Segmente der Mannesmann AG aus. Auffällig ist, dass die Frage der Feindlichkeit oder Nichtfeindlichkeit - ganz im Gegensatz zur Argumentation der IG Metall in 1997 - nicht im Vordergrund der Stellungnahmen von Betriebsrat und Gewerkschaft stand. Stattdessen wurden industriepolitische Forderungen gestellt: Die integrierte Telekommunikationsstrategie (Festnetz, Mobilfunk, Internet) solle erhalten bleiben, ebenso die von Klaus Esser eingeleitete Strategie zur Entwicklung der Mannesmann-Röhrenwerke, und der Börsengang von Engineering und Automotive (mittlerweile als „Atecs“ an Bosch-Siemens verkauft) solle durchgeführt werden.

Betriebsrat und Gewerkschaft stützten Klaus Essers Verteidigungsstrategie, die auf die Ablehnung des Übernahmeangebots an die Aktionäre bei gleichzeitigem Verzicht auf Kampf gegen das feindliche Übernahmeverfahren gerichtet war. Mitte November kam es zu einem Warnstreik von 500 Mannesmann-Angestellten, die gegen den feindlichen Übernahmeversuch demonstrierten. Gleichzeitig fand eine Konferenz der Betriebsräte aller Teilunternehmen statt. Gewerkschaft und Betriebs- 
räte sahen es als Erfolg an, dass sich die Arbeitnehmerinnen und Arbeitnehmer aus dem Telekommunikationsbereich ebenso lebhaft an den Protesten beteiligten wie jene aus den klassischen Bereichen. Der IG-Metall Vorsitzende Klaus Zwickel, der für die Arbeitnehmerseite im Aufsichtsrat von Mannesmann saß, bezog wiederholt gegen die Übernahme Stellung. Mannesmann sei ein kerngesundes Unternehmen mit hervorragenden Perspektiven, zudem mit einer besseren Strategie als Vodafone.

Gleichwohl: $\mathrm{Zu}$ besonders kämpferischen Reaktionen von Betriebsräten und Gewerkschaften kam es zwischen November 1999 und Februar 2000 nicht. Der Verzicht auf Kritik an dem Gebrauch des Instruments der feindlichen Übernahme als legitimem Mittel ist bemerkenswert, weil er in letzter Konsequenz die Dominanz der Aktionärsinteressen über andere im Unternehmen vertretenen Interessen legitimiert. Die grundsätzliche Akzeptanz der Aktionärsperspektive wurde in einer Rede Klaus Zwickels vor einer Mannesmann-Betriebsräteversammlung deutlich: „Wir werden die Aktionäre überzeugen, weil Mannesmann das bessere Konzept hat. [...] Das Beispiel Mannesmann wird - so hoffe ich - zeigen, daß die Solidarität der Betroffenen, die Weitsicht der Aktionäre und die Kompetenz der Fondsverwalter einen feindlichen Übernahmeversuch abwehren können."

\section{Die Rolle der Banken}

In der Auseinandersetzung zwischen Vodafone und Mannesmann hielt sich die Deutsche Bank als Hausbank auffällig zurück und spielte keine das deutsche Zielunternehmen beschützende Rolle. Der Grund für diese Zurückhaltung liegt in ihrer zunehmenden Fokussierung auf das Investmentbanking. Als These kann formuliert werden, dass die Deutsche Bank während des Übernahmekampfs zwischen Thyssen und Krupp erstmals aus der „Deutschland $\mathrm{AG}^{\text {“ }}$ ausbrach und der Wechsel vom „Hausbank-Paradigma“ auf das Investmentbanking bei der Deutschen Bank auf das Jahr 1997 zu terminieren ist.

Als Pirelli die Übernahme von Continental plante, votierte die Deutsche Bank, Hausbank bei Continental, zunächst für ein Zusammengehen beider Konzerne. Allerdings verfolgte der von der Deutschen Bank stammende, aber freilich auf die Unternehmensziele von Continental verpflichtete Aufsichtsratsvorsitzende Ulrich Weiß dabei durchaus Eigeninteressen: Im Vorstand der Deutschen Bank war er für das Italiengeschäft zuständig und erhoffte sich von einer gelungenen Übernahme einen Zugang zu dem von außen undurchdringlichen italienischen Industrienetzwerk. Hinzu kam noch, dass die Deutsche-Bank-Tochter Morgan Grenfell von Continental mit der Konstruktion einer Abwehrstrategie beauftragt worden war. Es kam zu schweren Konflikten im Aufsichtsrat von Continental, in deren Verlauf der Conti-Vorsitzende Urban seinen Rücktritt erklärte. Im Vorstand der Deutschen Bank setzten sich die Übernahmegegner durch, sodass die Deutsche 
Bank um 180 Grad umschwenkte und den Aufbau eines gegen die Übernahmeabsichten gerichteten Aktienblocks organisierte. Zweifellos hatte sich die Deutsche Bank wegen der widersprüchlichen Ziele, die sie verfolgte, blamiert.

Genauso verhielt es sich 1997 in der Auseinandersetzung zwischen Krupp und Thyssen. Einerseits war die Deutsche Bank im Thyssen-Aufsichtsrat vertreten und hatte das Unternehmen bei der Vorbereitung von Analystentreffen beraten. Andererseits unterstützte sie Krupp bei dem Versuch, den größeren und wirtschaftlich weniger angeschlagenen Thyssen-Konzern zu übernehmen. Die Deutsche Bank wollte sich in der Öffentlichkeit als moderne Investmentbank präsentieren. „Mir liegt sehr daran, daß dieser erste große Fall am Finanzplatz Deutschland ein Exempel setzt" ${ }^{8}$ äußerte das für das Investmentbanking zuständige Vorstandsmitglied Rolf Breuer damals, man wolle „Investmentbanking am Hochreck" ${ }^{\text {"9 }}$ demonstrieren. Erneut hatte sich die Deutsche Bank in Widersprüche verstrickt, und der öffentliche Protest gegen die Übernahmeattacke entlud sich zu einem Großteil gegen die Deutsche Bank. Im Vorstand der Deutschen Bank brach ein Konflikt zwischen Traditionalisten und Investmentbankern aus, in dem aber, anders als noch während des Abwehrkampfs der Continental AG, die Investmentbanker obsiegten.

Auch im Übernahmekampf zwischen Mannesmann und Vodafone war es wiederum - die Deutsche Bank, die enge Beziehungen zu dem Zielunternehmen hatte. In scharfem Kontrast zu den oben angedeuteten Vorgängen schaffte es die Deutsche Bank 1999/2000, sich aus dem Übernahmekampf herauszuhalten und jedwede Stellungnahme zu vermeiden. Eine besondere industriepolitische Rolle nahm die Deutsche Bank nicht mehr für sich in Anspruch. In ihrer Fokussierung auf das Investmentbanking wollen deutsche Großbanken demonstrieren, dass sie in ihren Tätigkeiten durch besondere Beziehungen zu „ihren“ deutschen Industrieunternehmen nicht restringiert werden. Logische Konsequenz ist ihr Rückzug aus dem Monitoring und mithin aus der „Beschützerrolle“ solcher Industrieunternehmen.

Nicht Hausbanken, sondern Investmentbanken traten im Abwehrkampf von Mannesmann in Erscheinung. Im Investmentbanking spielt die Einleitung und Begleitung von Akquisitionen eine entscheidende Rolle. Bei feindlichen Übernahmen treten Investmentbanken mehrfach in Erscheinung, nämlich als Berater der Bietergesellschaften wie auch bei der Entwicklung der Defensivstrategien der Zielunternehmen - und, nach erfolgter Übernahme, bei dem Management der dann folgenden Umstrukturierungen. Während der Auseinandersetzungen zwischen Mannesmann und Vodafone wurde Mannesmann von Morgan Stanley, Merrill Lynch und J.P. Morgan beraten, Goldman Sachs und Warburg Dillon Read arbeiteten Vodafone zu. Diese Aufzählung verdeutlicht die enorme amerikanische 
Dominanz im Investmentbanking, auch und sogar in einer Auseinandersetzung zwischen zwei europäischen Unternehmen.

\section{Der Ausgang des Übernahmekampfs}

Ab Mitte Januar 2000 verdichteten sich Anzeichen, eine Mehrheit der Mannesmann-Aktionäre werde an Vodafone verkaufen. Die Entscheidung im Übernahmekampf fiel, als Klaus Essers letzte und massivste Abwehrstrategie gründlich misslang: Die Suche nach einem „weißen Ritter“. Die Öffentlichkeit erfuhr, Mannesmann plane eine Zusammenlegung seines Kerngeschäfts mit den Telekomaktivitäten des französischen Mischkonzerns Vivendi. Kurz darauf schaltete sich auch Vodafone in die Verhandlungen mit Vivendi ein und brachte die Franzosen als Käufer von Orange ins Spiel.

Ende Januar waren die Fronten geklärt: Vivendi vereinbarte mit Vodafone, ein gemeinsames europäisches Internetportal betreiben zu wollen. Nach diesem Rückschlag war offenbar keine Mehrheit der Mannesmann-Aktionäre mehr zum Halten ihrer Anteile zu bewegen, denn ein gewichtiges Argument, das die Gegner der Fusion für sich in Anspruch genommen hatten - Vodafones Vernachlässigung des Internet - war damit gegenstandslos geworden. Am 3. Februar $2000 \mathrm{kam}$ es zur Einigung zwischen Vodafone und Mannesmann. Klaus Esser akzeptierte ein verbessertes Übernahmeangebot, dem zufolge Vodafone 50,5\% und Mannesmann $49,5 \%$ an der neuen Gesellschaft halten sollten. Anfang Februar 2001 stimmte der Aufsichtsrat, auch mit den Stimmen der Arbeitnehmerbank, der Fusion zu. Weitere Abwehrkämpfe - etwa unter Ausnutzung von Spitzfindigkeiten im deutschen Aktienrecht, mit denen auch eine Minderheit von Mannesmann-Aktionären die für die Fusion notwendige Abspaltung von Orange hätte erschweren können - wurden von der Arbeitnehmerbank nicht eingefordert.

\section{Ergebnisse}

Unsere Betrachtung zeigt, dass keine der in Abschnitt 2 genannten institutionellen Barrieren gegen feindliche Übernahmen - Eigentümerstrukturen, Hausbanken, Mitbestimmung, Unternehmens- und Aktienrecht - im Übernahmekampf zwischen Vodafone und Mannesmann eine verhindernde Wirkung entfalten konnte. War die feindliche Übernahme von Mannesmann ein Ausnahmefall oder ein paradigmatisches Ereignis (Jürgens/Rupp/Vitols 2000), das auf eine grundsätzliche Veränderung des deutschen Corporate Governance Systems hindeutet? In Abwägung der Argumente lautet unsere These, dass sich der institutionelle Kontext für deutsche Großunternehmen in der zweiten Hälfte der 1990er Jahre dahingehend verändert hat, dass feindliche Übernahmen großer deutscher Unternehmen 
prinzipiell möglich werden und sich damit neben dem Produkt-, Arbeits- und Aktienmarkt in Deutschland ein Markt für Unternehmenskontrolle herauskristallisiert. Nur eine begrenzte, tendenziell aber zunehmende Zahl deutscher Unternehmen ist diesem Markt ausgesetzt.

Im Falle Mannesmanns lag keine blockierte Eigentümerstruktur vor, die den Aufkauf einer Mehrheit der Anteile durch einen feindlichen Übernehmer hätte verhindern können. Wie in Abschnitt 2 gezeigt, war Mannesmann in dieser Hinsicht Ausnahme, nicht die Regel: Nur 11 der 100 größten deutschen Unternehmen wiesen 1998 einen Streubesitzanteil von mehr als 75\% aller Aktien auf (Monopolkommission 2000, S. 259-265). Das bedeutet, dass nur eine Minderheit der deutschen Großunternehmen dem „freien“, also auch gegen den Willen der Manager der Zielunternehmen funktionsfähigen Markt für Unternehmenskontrolle ausgesetzt ist. Allerdings kann prognostiziert werden, dass sich die Zahl der feindlich übernehmbaren Großunternehmen vergrößern wird und in naher Zukunft deutliche Schritte in Richtung Entflechtung der „Deutschland AG“ stattfinden werden. Ab Januar 2002 greift die Änderung des Körperschaftssteuergesetzes, mit der die Steuerbefreiung von Gewinnen aus Beteiligungsveräußerungen auch auf inländische Unternehmensbeteiligungen ausgedehnt wird (Höpner 2000). Die Finanzunternehmen des deutschen Verflechtungszentrums (Beyer 1998, S. 158), insbesondere Deutsche Bank, Dresdner Bank, Allianz und Münchener Rück, haben angekündigt, diese Möglichkeit nutzen und sich von Beteiligungen an Industrieunternehmen trennen $\mathrm{zu}$ wollen.

Damit ist zwar nicht gesagt, dass diese Aktienpakete komplett in Streubesitz transformiert werden. Neben der Platzierung dieser Anteile an der Börse besteht die Möglichkeit, sie im Block an einen Abnehmer zu veräußern. Auch in diesem Falle werden die betroffenen Unternehmen in Zukunft verstärkt dem Markt für Unternehmenskontrolle ausgesetzt sein, denn strategische Aktienpakete verwandeln sich in finanzorientierte (Beyer 1998). Durch den Verkauf von aus strategischen Motiven gehaltenen Aktienblocks an Abnehmer, für die die entsprechenden Pakete den Charakter von Finanzanlagen haben, steigt die Anzahl der Aktien, die für einen feindlichen Übernehmer prinzipiell erwerbbar sind. Auch die zunehmende Institutionalisierung des Aktienbesitzes wirkt tendenziell treibend auf den Aktienanteil, der für feindliche Übernehmer prinzipiell zugänglich ist.

Als entscheidender Anstoß für das Entstehen eines Marktes für Unternehmenskontrolle in Deutschland muss der Strategiewechsel deutscher Großbanken gewertet werden. Insbesondere die Deutsche Bank hat gute Chancen, sich nach der Akquisition von Bankers Trust zu einer der großen internationalen Investmentbanken neben Goldman Sachs, Morgan Stanley und Merrill Lynch zu entwickeln. $\mathrm{Zu}$ den Kerngeschäften von Investmentbanken gehört die Beratung bei Fusionen und Übernahmen, ein Geschäft, das zu den engen, loyalen Beziehungen zu deutschen Industrieunternehmen in Widerspruch steht. In diesem Konflikt haben sich die Investmentbanker in den Vorständen deutscher Großbanken - nicht nur bei 
der Deutschen Bank, sondern auch bei (ehemaliger) Dresdner Bank und Commerzbank - eindeutig durchgesetzt. Aus diesen Entwicklungen kann gefolgert werden, dass deutsche Großbanken in Zukunft nicht mehr als Verhinderer feindlicher Übernahmen auftreten werden. Sie werden sich aus dem Monitoring von Industrieunternehmen weiter zurückziehen und kredit- und industriepolitische Interessen hintanstellen, wenn sie als Manager feindlicher Übernahmen oder als Entwickler von Defensivstrategien in Anspruch genommen werden.

Unternehmensbezogene Daten deuten darauf hin, dass das Monitoring der Banken bei deutschen Industrieunternehmen in den späten 1990er Jahren bereits stark rückläufig ist. Noch 1996 hatte die Deutsche Bank in 29 Aufsichtsräte der 100 größten deutschen Unternehmen Vorstandsmitglieder entsandt. ${ }^{10}$ Nur zwei Jahre später, 1998, ist diese Zahl auf 17 gesunken. In einem Sample der 40 größten börsennotierten Nichtfinanzunternehmen hat sich der Anteil von Unternehmen, bei denen der Aufsichtsratsvorsitzende ein Vertreter eines Finanzunternehmens ist, im Laufe der 1990er Jahre von etwa 40 auf gut 20 Prozent fast halbiert. Hier muss von einem rapiden Wechsel in den Kontrollstrukturen großer deutscher Unternehmen gesprochen werden. In ihren im März 2001 vorgestellten Corporate-Governance-Grundsätzen hat die Deutsche Bank sogar angekündigt, künftig überhaupt keine Aufsichtsratsvorsitze von Nichtfinanzunternehmen mehr übernehmen zu wollen (Deutsche Bank 2001). Jürgen Beyer und Paul Windolf haben herausgearbeitet, dass für deutsche Verflechtungsstrukturen ein hohes Maß an Kongruenz zwischen Personal- und Kapitalverflechtung typisch ist (Beyer 1998; Windolf/Beyer 1995; Windolf/Nollert 2001). Die in den 1990er Jahren rückläufige Personalverflechtung muss deshalb als Frühindikator einer bevorstehenden Kapitalentflechtung interpretiert werden.

Mitbestimmung und Gewerkschaften traten im Fall Mannesmann nicht als grundsätzliche Kritiker feindlicher Übernahmen auf. Vergleicht man die Übernahmeversuche bei Thyssen und bei Mannesmann, dann zeigt sich, dass ordnungspolitische Argumentationen durch industriepolitische Argumente und Forderungen ausgetauscht wurden. Mit aller Vorsicht wollen wir die These formulieren, dass die deutschen Gewerkschaften auch in zukünftigen Übernahmeauseinandersetzungen bei der grundsätzlichen Akzeptanz von Übernahmeangeboten als legitimem Instrument wirtschaftlichen Handelns bleiben werden. Allerdings scheint eine Prognose in dieser Frage schwieriger als bei den abzusehenden Entflechtungstendenzen oder der Geschäftsstrategie deutscher Großbanken. Möglicherweise ist die Reaktion der IG Metall als experimentelle, unter Ungewissheit zustande gekommene Reaktion zu werten, und damit revidierbar. Für diese Sicht spricht, dass VodafoneChef Chris Gent nach erfolgter Übernahme keines der Versprechen, auf deren Grundlage die Arbeitnehmerbank im Mannesmann-Aufsichtsrat ihre Zustimmung gab, einhielt: Die Bereiche Maschinenbau und Automobilzulieferung wurden nicht

10 Diese und die nachfolgenden Informationen stammen aus der Unternehmensdatenbank am Max-Planck-Institut für Gesellschaftsforschung. 
an die Börse gebracht, sondern als „Atecs“ an den Meistbietenden (Bosch-Siemens) verkauft; der Konzernzentrale in Düsseldorf kommt, anders als vorher angedeutet, keine hohe Bedeutung mehr zu; und der Traditionsname Mannesmann wurde nicht beibehalten, sondern das Mobilfunknetz in „Vodafone D2“ umbenannt. Auf den ersten Blick führte die feindliche Übernahme von Mannesmann tatsächlich zu einem Bruch impliziter Verträge und damit zur Zerstörung von Vertrauen (Shleifer/Summers 1988). Es kann nicht ausgeschlossen werden, dass deutsche Gewerkschaften nach diesen Erfahrungen auf künftige Übernahmefälle ablehnender reagieren werden, zumal das Risiko von Arbeitsplatzverlusten bei Mannesmann aufgrund seiner Zugehörigkeit zum boomenden Telekommunikationssektor gering war.

Gegen diese Annahme spricht allerdings, dass solche Reaktionen nur geringe Aussichten auf Erfolg hätten. Im Zusammenspiel mit den anderen besprochenen institutionellen Mechanismen mögen Gewerkschaften als Übernahmeverhinderer eine Rolle gespielt haben; die Macht, im Umfeld der frühen 2000er Jahre feindliche Übernahmen allein zu verhindern, haben deutsche Gewerkschaften nicht. Bedacht werden sollte auch, dass die organisierten Belegschaften bei nationalen Übernahmekämpfen durchaus unterschiedliche produktionsbezogene Interessen haben. ${ }^{11}$ Schon während des Übernahmekampfs zwischen Thyssen und Krupp im Jahr 1997 musste die IG Metall die Erfahrung machen, dass es unmöglich war, Interessenvertreter der Krupp-Belegschaft zum Protest gegen den feindlichen Übernahmeversuch Crommes zu bewegen. Der These rom Vertrauensbruch im Zuge der Mannesmann-Übernahme ist entgegenzuhalten, dass der Verkauf von Atecs an Bosch-Siemens unter Einbeziehung der Mitbestimmung zustande kam. Auch in der IG Metall gab es Stimmen, die den Verkauf an Bosch-Siemens oder ThyssenKrupp präferierten. Wenn es aus Arbeitnehmerperspektive einen Vertrauensbruch gab, fand er nicht zuletzt in den eigenen Reihen statt. Auch die unappetitlichen Vorgänge um die astronomischen Abfindungen Klaus Essers, seines Stabs und sogar des Aufsichtsratsvorsitzenden Joachim Funk sprechen gegen die Vertrauensbruch-These: Die Übernommenen haben - offenbar mit Zustimmung Klaus Zwickels, der Mitglied im Aufsichtsratsausschuss für Vorstandsangelegenheiten war - schlimmer gewütet, als man es von den Übernehmern hätte befürchten können.

Vor allem aber fällt auf, dass Mitbestimmung und Gewerkschaften sowohl bei Continental wie bei Thyssen und auch bei Mannesmann die grundsätzliche Verteidigungsstrategie der angegriffenen Unternehmen unterstützen. Da als sicher gelten kann, dass auch künftig die Angegriffenen auf der Grundlage des Finanzinteresses der Aktionäre argumentieren werden, bleibt es unter dem Strich bei der vorsichtigen These, dass die Hinnahme feindlicher Übernahmen durch die deutschen Gewerkschaften von Dauer sein wird.

11 Siehe zur Unterscheidung zwischen klassen- und produktionsbezogenen Interessen (Streeck 1992a, S. 96). 
Auch die Institution der Betriebsratsmitbestimmung bei Mannesmann wirkte nicht abschreckend auf den ausländischen feindlichen Übernehmer. In Abschnitt 2 haben wir gezeigt, dass die 1999 beschlossene Konzernspaltung nicht gegen die Mitbestimmungsträger durchgesetzt werden musste, sondern von den Arbeitnehmervertretern sogar vorangetrieben wurde. Auch Internationalisierungsentscheidungen fallen in Großunternehmen in der Regel im Konsens mit der Arbeitnehmerseite. Insgesamt scheint sich in den 1990er Jahren ein Trend verstärkt zu haben, der bereits in den 1980er Jahren beschrieben wurde: Der Trend zu Versachlichung, Professionalisierung und Produktivitätsorientierung der Mitbestimmung (Mitbestimmungskommission 1998, S. 76 f; Müller-Jentsch/Seitz 1998, S. 367). „Unterschiede zwischen Interessenvertretung einerseits und Beteiligung an den Leitungsfunktionen des Unternehmens andererseits verschwimmen“, stellte die gemeinsame Mitbestimmungskommission von Bertelsmann-Stiftung und Hans Böckler Stiftung fest (Mitbestimmungskommission 1998, S. 76 f.). Eine solche, am "Co-Management" orientierte Mitbestimmung wird auch von ausländischen Unternehmen offenbar nicht mehr als „Giftpille“ gegen Übernahmen interpretiert.

Hinsichtlich der Besonderheiten des deutschen Aktien- und Unternehmensrechts fällt der Befund eindeutig aus: Der Status Quo des Unternehmensrechts hat die Übernahme von Mannesmann nicht verhindert, und die Verabschiedung ,übernahmeverhindernder" Bestimmung kann für die Zukunft ausgeschlossen werden. Durch das 1998 verabschiedete Kapitalaufnahmeerleichterungsgesetz (KapAEG) wurde Konzernobergesellschaften freigestellt, nach den Regeln des Handelsgesetzbuchs (HGB) oder alternativ nach den internationalen International Accounting Standards (IAS) oder den General Accepted Accounting Principles (US-GAAP) zu bilanzieren. Bereits im Herbst 1999 bilanzierten 33 der 100 größten deutschen Unternehmen nach internationalen Regeln (Hassel et al. 2000). Diese kapitalmarktorientierten Maßnahmen erhöhen die Fähigkeit potenzieller Übernehmer, die Werthaltigkeit deutscher Unternehmen von außen einzuschätzen. Durch das Gesetz zur Kontrolle und Transparenz im Unternehmensbereich (KonTraG) wurden die Rechte von Minderheitsaktionären gestärkt, Stimmrechtsbeschränkungen wurden verboten. Weitergehende Reformen zur Stärkung der Rechte von Kleinaktionären und zum Ausbau der Berichtspflichten der Manager gegenüber den Kontrollorganen befinden sich in Vorbereitung („KonTraG 2“) und können mit hoher Wahrscheinlichkeit im Konsens mit den Gewerkschaften verabschiedet werden (Bolt 2000; Köstler 2000). Allem voran aber ist in Deutschland keine Übernahmeregulierung in Sicht, deren Ziel es wäre, feindliche Übernahmen zu bremsen. Auch die Einräumung von Vorratsbeschlüssen durch die Hauptversammlung macht das geplante Übernahmegesetz nicht zu einem Übernahmeverhinderungsgesetz.

Unser Fazit lautet deshalb: Nahezu alle Veränderungen, die in den 1990er Jahren stattfanden, legen die Prognose nahe, dass Mannesmann kein Einzelfall bleiben wird. Stattdessen hat ein tiefgreifender Wandel des deutschen Corporate 
Governance Systems stattgefunden, der einen Markt für Unternehmenskontrolle entstehen lässt. Dieses neue institutionelle Umfeld verändert das Anreizsystem, auf dessen Grundlage Manager ihre Entscheidungen fällen. Wo „klassische“ Übernahmebarrieren fallen und keine blockierten Eigentümerstrukturen vorliegen, bleibt als einziger Übernahmeschutz ein hoher Aktienkurs. Vor diesem Hintergrund kann mit der Ausweitung von „Shareholder Value"-Strategien großer deutscher Unternehmen gerechnet werden. Die Passgüte von Schlagworten wie „stakeholderorientiert", „insiderorientiert" oder gar „bankenorientiert" wird zur Beschreibung des „deutschen Modells“ immer fragwürdiger.

Konvergenzschritte in Richtung auf das angelsächsische Modell im Sinne der Einführung eines eingliedrigen Board-Systems oder gar einer Abschaffung der Mitbestimmung sind nicht zu erwarten. Gleichwohl müssen Stabilitätshypothesen, die keine radikalen Veränderungen des deutschen Corporate Governance Systems nahelegen, als falsifiziert angesehen werden. Das „deutsche Modell“ durchläuft einen Prozess der Hybridisierung (Jackson 2001; Vitols 2000), in dem die institutionelle Absicherung der Partizipationsmöglichkeiten von Arbeitnehmern bestehen bleiben wird - die sich ihrerseits in der Abwägung ihrer Handlungsalternativen mit den Herausforderungen internationaler Kapitalmärkte, inklusive eines „freien“ Markts für Unternehmenskontrolle, konfrontiert sehen.

\section{Literatur}

Albert, Michel, 1992: Kapitalismus contra Kapitalismus, Frankfurt a.M.: Campus Verlag.

Baums, Theodor, 1993: Hostile Takeovers in Germany. A Case Study on Pirelli vs. Continental AG. Arbeitspapier 3/93, Osnabrück: Institut für Handels- und Wirtschaftsrecht, Universität Osnabrück.

Baums, Theodor und Christian Fraune, 1995: Institutionelle Anleger und Publikumsgesellschaft: Eine empirische Untersuchung, in: Die Aktiengesellschaft, Heft 3, S. 97-112.

Beyer, Jürgen, 1998: Managerherrschaft in Deutschland? 'Corporate Governance' unter Verflechtungsbedingungen, Opladen: Westdeutscher Verlag.

Bhagat, Sanjai, Andrei Shleifer und Robert W. Vishney, 1990: Hostile Takeovers in the 1980s: The Return to Corporate Specialization, in: M.N. Baily und C. Winston (Hrsg.), Brookings Papers on Economic Activity: Microeconomics 1990, Washington DC: Brookings Institution, $S, 1-84$.

Bittlingmayer, George, 1998: The Market for Corporate Control (Including Takeovers), in: Boudewijn Bouckaert und Gerrit De Geest (Hrsg.), Encyclopedia of Law and Economics, Aldershot: Edward Elgar.

Bolt, Marie, 2000: Stellungnahme des DGB zum Fragenkatalog der Regierungskommission „Corporate Governance - Unternehmensführung - Unternehmenskontrolle - Modernisierung des Aktienrechts", Berlin: Deutscher Gewerkschaftsbund.

Davis, Gerald F., Kristina A. Diekmann und Catherine H. Tinsley, 1994: The Decline and Fall of the Conglomerate Firm in the 1980s: The De-Institutionalization of an Organizational Form, in: American Sociological Review 59, S. 547-570. 
Davis, Gerald F. und Gregory E. Robbins, 2001: The Fate of the Conglomerate Firm in the United States, in: Walter W. Powell und Daniel L. Jones (Hrsg.), How Institutions Change, Chicago, IL: University of Chicago Press.

Davis, Gerald F. und Suzanne K. Stout, 1992: Organization Theory and the Market for Corporate Control: A Dynamic Analysis of the Characteristics of Large Takeover Targets, 1980-1990, in: Administrative Science Quarterly 37, S. 605-633.

Deutsche Bank, 2001: Die Corporate Governance-Grundsätze der Deutschen Bank. Corporate Governance als Teil des Selbstverständnisses der Deutschen Bank, Frankfurt a.M.: Deutsche Bank AG.

Dietrich, Michael, 1994: National Patterns of Corporate Restructuring: Mergers and Joint Ventures in the European Community, in: Robert Delorme und Kurt Dopfer (Hrsg.), The Political Economy of Diversity. Evolutionary Perspectives on Economic Order and Disorder, Aldershot: Edward Elgar, S. 149-165.

Erker, Paul, 1996: Wachsen im Wettbewerb. Eine Zeitgeschichte der Continental Aktiengesellschaft (1971-1996) anläßlich des 125jährigen Firmenjubiläums, Düsseldorf: Econ.

Franks, Julian und Colin Mayer, 1995: Hostile Takeovers in the UK and the Correction of Managerial Failure, in: London Business School, Institute of Finance and Accounting Working Paper, No. 156.

Franks, Julian und Colin Mayer, 1998: Bank Control, Takeovers, and Corporate Governance in Germany, in: Klaus J. Hopt et al. (Hrsg.), Comparative Corporate Governance. The State of the Art and Emerging Research, Oxford: Oxford University Press, S. 641-657.

Gerke, Wolfgang, Hendrik Garz und Marc Oerke, 1995: Die Bewertung von Unternehmensübernahmen auf dem deutschen Aktienmarkt, in: Zeitschrift für betriebswirtschaftliche Forschung 47, S. 805-820.

Graf, Jürgen, Christian Lenke und Stefan Schießer, 1997: Die Umsetzung des Shareholder-Value-Konzepts durch die DAX-Unternehmen. Studie der SGZ-Bank AG, Frankfurt a.M./ Karlsruhe: SGZ-Bank.

Hall, Peter A. und David Soskice, 2001: An Introduction to Varieties of Capitalism, in: Peter A. Hall und David Soskice (Hrsg.), Varieties of Capitalism: Institutional Foundations of Comparative Advantage, Cambridge: Cambridge University Press.

Hassel, Anke et al,, 2000: Zwei Dimensionen der Internationalisierung: Eine empirische Analyse deutscher Grossunternehmen, in: Kölner Zeitschrift für Soziologie und Sozialpsychologie, 52. Jg., S. 500-519.

Höpner, Martin, 2000: Unternehmensverflechtung im Zwielicht. Hans Eichels Plan zur Auflösung der Deutschland AG, in: WSI-Mitteilungen 53.

Höpner, Martin, 2001: Corporate Governance in Transition. Ten Empirical Findings on Shareholder Value and Industrial Relations in Germany. MPIfG Discussion Paper 05-2001, Köln: Max-Planck-Institut für Gesellschaftsforschung.

Jackson, Gregory, 2001: Organizing the Firm: The Evolution of Corporate Governance in Germany and Japan. Dissertation, New York, NY: Columbia University.

de Jong, Henk W., 1992: Der Markt für Unternehmenskontrolle. Eine historische, theoretische und empirische Betrachtung, in: Helmut Gröner (Hrsg.), Der Markt für Unternehmenskontrollen, Berlin: Duncker \& Humblot, S. 141-159.

de Jong, Henk W., 1997: The Governance Structure and Performance of Large European Corporations, in: The Journal of Management and Governance 1, S. 5-27.

Jürgens, Ulrich, Joachim Rupp und Katrin Vitols, 2000: Corporate Governance und Shareholder Value in Deutschland. Nachtrag von Ulrich Jürgens: Nach dem Fall von Mannesmann Paper revisited. WZB Discussion Paper FS II 00-202, Berlin: Wissenschaftszentrum Berlin.

Köstler, Roland, 2000: Anforderungen der Arbeitnehmer an eine effektive Unternehmensüberwachung. Papier für die Konferenz „Institutioneller Wandel in den industriellen Bezichungen", 8.-9.12.2000, Max-Planck-Institut für Gesellschaftsforschung, Köln. 
Manne, Henry, 1965: Mergers and the Market for Corporate Control, in: Journal of Political Economy 73, S. 110-120.

Mannesmann, 2000: Mannesmann bietet mehr Wert heute, mehr Wert morgen und weniger Risiko. Nein zum Vodafone-Angebot, Düsseldorf: Mannesmann AG.

Mitbestimmungskommission, 1998: Mitbestimmung und neue Unternehmenskulturen. $\mathrm{Bi}$ lanz und Perspektiven, Gütersloh: Verlag Bertelsmann Stiftung.

Monopolkommission, 1980: Fusionskontrolle bleibt vorrangig. Drittes Hauptgutachten der Monopolkommission, Baden-Baden: Nomos Verlagsgesellschaft.

Monopolkommission, 2000: Wettbewerbspolitik in Netzwerkstrukturen. Dreizehntes Hauptgutachten der Monopolkommission, Baden-Baden: Nomos Verlagsgesellschaft.

Müller-Jentsch, Walther und Beate Seitz, 1998: Betriebsräte gewinnen Konturen. Ergebnisse einer Betriebsräte-Befragung im Maschinenbat, in: Industrielle Beziehungen 5, S. 361387.

Nuttall, Robin, 1999: An Empirical Analysis of the Effects of the Threat of Takeover on UK Company Performance, Nuffield College, University of Oxford Working Paper, No. 5.

Shleifer, Andrei und Lawrence H. Summers, 1988: Breach of Trust in Hostile Takeovers, in: Alan J. Auerbach (Hrsg.), Corporate Takeovers: Causes and Consequences, Chicago, IL: The University of Chicago Press, S. 33-68.

Shleifer, Andrei und Robert W. Vishny, 1996: A survey of corporate governance. NBER working paper series; Working Paper No. 5554, Cambridge, MA: National Bureau of Economic Research.

Streeck, Wolfgang, 1992a: Interest Heterogenity and Organizing Capacity: Two Class Logics of Collective Action?, in: Wolfgang Streeck (Hrsg.), Social Institutions and Economic Performance. Studies of Industrial Relations and Economic Performance, London: Sage, S. 176-194.

Streeck, Wolfgang, 1992b: On the Institutional Conditions of Diversified Quality Production, in: Egon Matzner und Wolfgang Streeck (Hrsg.), Beyond Keynesianism. The Socio-Economics of Production and Full Employment, Aldershot: Edward Elgar, S. 21-61.

Streeck, Wolfgang, 1997: Beneficial Constraints: On the Economic Limits of Rational Voluntarism, in: J. Rogers Hollingsworth und Robert Boyer (Hrsg.), Contemporary Capitalism: The Embeddedness of Institutions, Cambridge, UK: Cambridge University Press, S. 197 219.

Vitols, Sigurt, 2000: The Reconstruction of German Corporate Governance: Reassesing the Role of Capital Market Pressures. Papier für das „First Annual Meeting of the Research Network on Corporate Governance", Juni 23-24, 2000, Wissenschaftszentrum Berlin.

Vodafone, 1999: Offer for Mannesmann AG. Vodafone AirTouch and Mannesmann: Better Together, London: Vodafone Air'Touch.

Vogel, Dieter, 1997: „Konsens ist kein Schimpfwort“. Ein Zeit-Gespräch mit Thyssen-Chef Dieter Vogel, in: Die Zeit, Nr. 17, 18.4.1997, S. 25.

Windolf, Paul, 1994: Die neuen Eigentümer. Eine Analyse des Marktes für Unternehmenskontrolle, in: Zeitschrift für Soziologie, 23. Jg., S. 79-92.

Windolf, Paul und Jürgen Beyer, 1995: Kooperativer Kapitalismus - Unternehmensverflechtungen im internationalen Vergleich, in: Kölner Zeitschrift für Soziologie und Sozialpsychologie, 47. Jg., S. 1-36.

Windolf, Paul und Michael Nollert, 2001: Institutionen, Interessen, Netzwerke. Unternehmensverflechtung im internationalen Vergleich, in: Politische Vierteljahresschrift, 42. Jg, S. 51-78. 